\title{
IRF5 distinguishes severe asthma in humans and drives Th1 phenotype and airway hyperreactivity in mice
}

\author{
Timothy B. Oriss, ${ }^{1}$ Mahesh Raundhal,,,2 Christina Morse,, ${ }^{1}$ Rachael E. Huff,' Sudipta Das, ${ }^{1}$ \\ Rachel Hannum, ${ }^{1}$ Marc C. Gauthier, ${ }^{1,3}$ Kathryn L. Scholl,, ${ }^{1}$ Krishnendu Chakraborty, ${ }^{1}$ \\ Seyed M. Nouraie, ${ }^{1}$ Sally E. Wenzel, ${ }^{1,2,3}$ Prabir Ray, ${ }^{1,2,3}$ and Anuradha Ray,2,3 \\ 'Division of Pulmonary, Allergy, and Critical Care Medicine, Department of Medicine, ${ }^{2}$ Department of Immunology, and \\ ${ }^{3}$ University of Pittsburgh Asthma Institute at University of Pittsburgh Medical Center, University of Pittsburgh School of \\ Medicine, Pittsburgh, Pennsylvania, USA.
}

Severe asthma (SA) is a significant problem both clinically and economically, given its poor response to corticosteroids (CS). We recently reported a complex type 1-dominated (IFN- $\gamma$-dominated) immune response in more than $50 \%$ of severe asthmatics despite high-dose CS treatment. Also, IFN- $\gamma$ was found to be critical for increased airway hyperreactivity (AHR) in our model of SA. The transcription factor IRF5 expressed in M1 macrophages can induce a Th1/Th17 response in cocultured human T cells. Here we show markedly higher expression of IRF5 in bronchoalveolar lavage (BAL) cells of severe asthmatics as compared with that in cells from milder asthmatics or healthy controls. Using our SA mouse model, we demonstrate that lack of IRF5 in lymph node migratory DCs severely limits their ability to stimulate the generation of IFN- $\gamma$ - and IL-17-producing $\mathrm{CD}^{+} \mathrm{T}$ cells and IRF5${ }^{-1-}$ mice subjected to the SA model displayed significantly lower IFN- $\gamma$ and IL-17 responses, albeit showing a reciprocal increase in Th2 response. However, the absence of IRF5 rendered the mice responsive to $\mathrm{CS}$ with suppression of the heightened Th2 response. These data support the notion that IRF5 inhibition in combination with CS may be a viable approach to manage disease in a subset of severe asthmatics.

Conflict of interest: The authors have declared that no conflict of interest exists.

Submitted: September 30, 2016 Accepted: April 18, 2017 Published: May 18, 2017

\section{Reference information:} JCI Insight. 2017;2(10):e91019. https://doi.org/10.1172/jici. insight.91019.

\section{Introduction}

Asthma in its many forms $(1,2)$ has a notably high prevalence in Western society. Fifty percent of asthmatics clinically diagnosed as mild-moderate (M/MOD) ( $~ 90 \%$ of all asthma) display atopy driven by a type 2 immune response in whom disease symptoms are well controlled by low-dose inhaled corticosteroid (CS) (3). However, a small subset of asthmatics (approximately 5\%-10\%), clinically classified as severe according to the European Respiratory Society and American Thoracic Society (ERS/ATS) guidelines (4), do not respond well to even high doses of inhaled or oral CS therapy and therefore generally have significant morbidity and overall poor quality of life (5). By definition, these individuals are typically treated with CS, but also require a second controller, such as a $\beta 2$ agonist, a leukotriene modifier, or systemic steroids. Still, in spite of this approach, asthma symptoms in some remain uncontrolled, resulting in frequent and often significant exacerbations. Importantly as well, the overall economic impact of this small proportion of cases nonetheless accounts for over half of the $\$ 56$ billion attributed to asthma in the United States alone according to the Centers for Disease Control and other sources $(3,6-8)$. Thus, lack of CS efficacy in these individuals represents a complex problem in terms of both clinical management and health care costs.

A major factor emerging for the immunologic basis of non-type 2 severe asthma (SA), as described previously by us, appears to be the production of high levels of IFN- $\gamma$ from CD4 ${ }^{+}$T cells $(9,10)$, which was also shown previously at the RNA expression level (11-14). IFN- $\gamma$ likely has myriad downstream effects such as upregulation of several hundred genes in airway epithelial cells, release of chemokines resulting in recruitment of additional inflammatory cells, and increase in iNOS expression causing increased exhaled nitric oxide (FeNO) (15-19). We have also recently shown that IFN- $\gamma$ is critical for airway hyperresponsiveness (AHR) in a model of SA established by us that mimics the immune response and poor response to CS in human SA (9). While more studies are needed to determine how a high level of IFN- $\gamma$ promotes 
AHR, an inverse association has been noted in multiple studies between IFN- $\gamma$ and the expression of the antiprotease, secretory protease leukocyte inhibitor (SLPI) $(9,20,21)$. The airways of WT mice subjected to the SA model with increased expression of Ifng showed significantly lower expression of Slpi compared with those of similarly treated $I F N G^{-1-}$ mice (9). Paired analysis of IFNG and SLPI expression in the airway cells of human severe asthmatics revealed an inverse relationship (9). SLPI inhibits multiple leukocyte serine proteases, which include the mast cell proteases chymase and tryptase, and neutrophil elastase. Tryptase was previously shown to induce AHR by activating protease-activated receptor 2 (PAR-2), which is expressed on airway smooth muscle cells and has been implicated in bronchoconstriction through release of neurokinins from afferent neurons in the airways (22). Also, human mast cell-derived tryptase can degrade bronchodilating neuropeptides (23), which would be facilitated by inhibition of SLPI expression by IFN- $\gamma(9)$. Collectively, these studies suggest that controlling expression of IFN- $\gamma$ may be a novel and potentially beneficial avenue for therapeutic intervention in SA.

The transcription factor IRF5 presented itself as an attractive candidate for investigation, given that it was shown to promote the transcription of multiple inflammation-associated genes (24). Overexpression of IRF5 in human macrophages promoted transcription of IL6, IL12, and IL23p19, leading to increased expression of IFN- $\gamma$ and IL-17 in cocultured human $\mathrm{CD}^{+} \mathrm{T}$ cells (25). We reasoned that our murine model of SA offered an opportunity to test a direct mechanistic link between environmental factors and the SA phenotype via IRF5. As detailed previously, pathogens may play a role in the establishment of SA through the generation of intracellular second messengers, which initially prompted us to evaluate the potential of the bacterial product, cyclic diguanosine monophosphate (c-di-GMP), as a component of the SA model (9). A number of bacterial species belonging to the genera Streptococcus and Haemophilus among others have been reported to both produce c-di-GMP and to be associated with SA in humans $(26,27)$. Furthermore, when used as a mucosal adjuvant, c-di-GMP was shown to result in a mixed Th1/Th17 immune response accompanied by a Th2 response, with production of antigen-specific IgG2a (type 1 associated) (28). Most importantly, in terms of relevance to immune-mediated pathophysiology, c-di-nucleotides like c-di-GMP have been associated with IRF5 activation (29). This occurs via c-di-GMP binding to the intracellular pattern recognition receptor, stimulator of interferon genes (STING), leading to activation of the kinase TBK1, resulting in phosphorylation of IRF3, as well as of IRF5 and IRF7 (29-32). Sustained STING activation has been associated with overproduction of cytokines (33).

In the context of our finding of an IFN- $\gamma$ bias in more than $50 \%$ of severe asthmatics (9), we investigated whether IRF5 expression is higher in SA and whether it is involved in promoting AHR in our mouse model of SA. Herein we show a significantly higher level of expression of IRF5 in bronchoalveolar lavage (BAL) cells of severe asthmatics as compared with that in cells from milder asthmatics or healthy controls, and also show a role for IRF5 in promoting AHR in our murine model of SA (9). Using our SA model, we show that lack of IRF5 in lymph node migratory DCs (migDCs) severely limits their ability to stimulate generation of IFN- $\gamma$ - and IL-17-producing $\mathrm{CD}^{+} \mathrm{T}$ cells and $I R F 5^{-1-}$ mice subjected to the SA model have significantly lower lung IFN- $\gamma$ and IL-17 levels. Although the IL-5 response and airway eosinophilia were not inhibited by CS in WT mice subjected to the SA model, in the IRF5 ${ }^{-1-}$ mice, which actually displayed a higher Th2 response, lung Th2 cytokine levels, serum IgE levels, and airway eosinophilia were all suppressed by CS. These data support the notion that IRF5 inhibition in combination with CS may be a viable therapeutic approach for the management of disease in a subset of severe asthmatics.

\section{Results}

Increased IRF5 expression in BAL cells in human SA. IRF5 emerged as a candidate gene for association with SA given its capacity to induce $\mathrm{CD}^{+} \mathrm{T}$ cell IFN- $\gamma$ secretion via expression of cytokines such as IL-12 in antigen-presenting cells (APCs) in vitro (25). High expression of IFN- $\gamma$ is now recognized as a cardinal feature in a subset of SA subjects (9-14), which we have recapitulated in our recently developed murine model (9). Based on these findings, we examined IRF5 mRNA expression in human BAL cells, which include not only lymphocytes but also a very high percentage of macrophages and fewer DCs. As shown in Figure 1A, we observed a significantly higher IRF5 expression in human BAL cells collected from severe asthmatics as compared with that in cells from mild asthmatics or healthy controls. A key feature of our SA model is the application of the bacterially derived second messenger c-di-GMP to activate IRF5 (29-32) and when administered in combination with house dust mite antigen (HDM), a high Th1/Th17 immune response is induced along with a low Th2 response (9). The model for M/MOD asthma employs HDM alone, which 


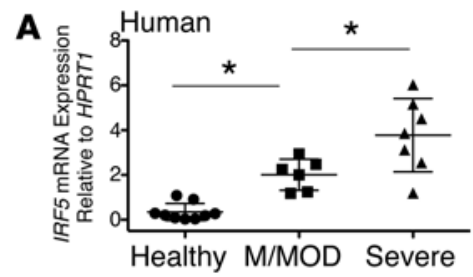

B
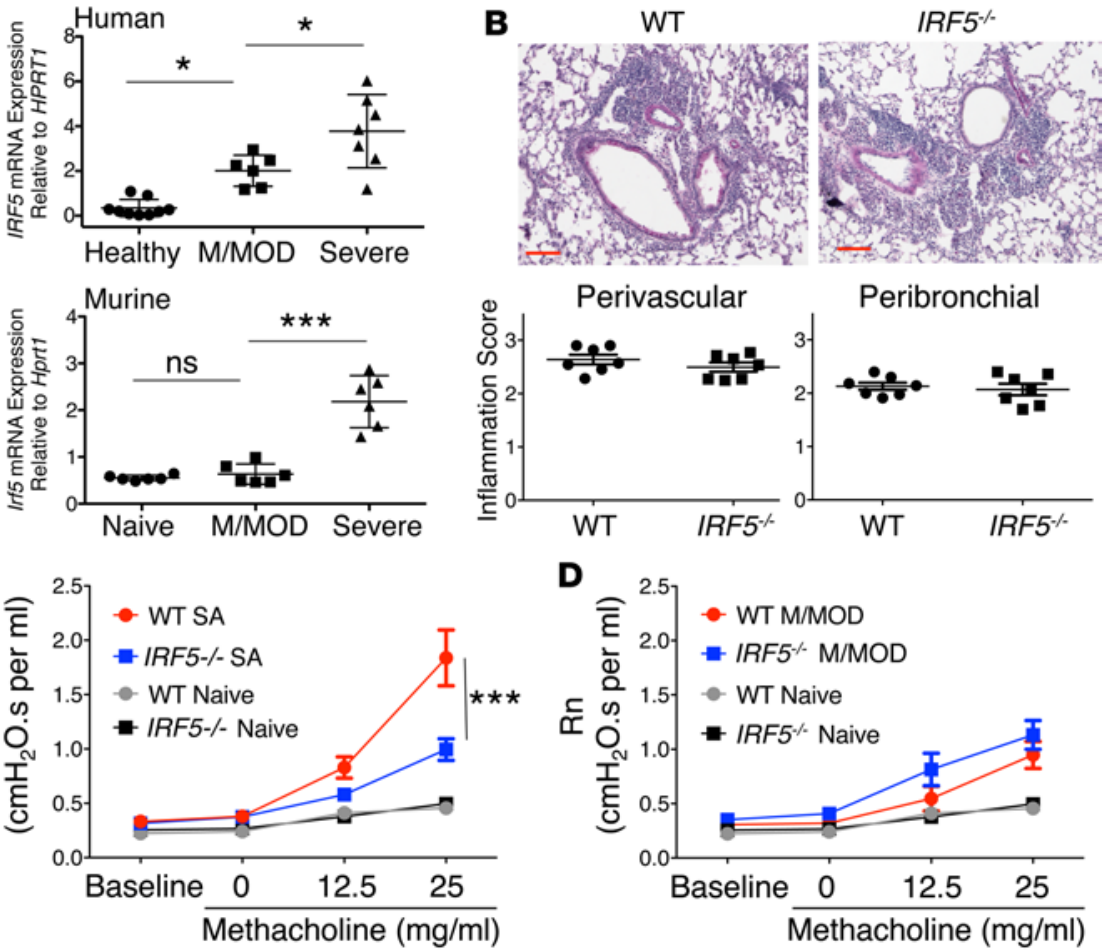

Perivascular

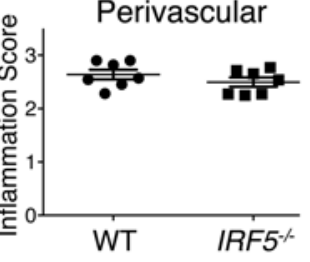

Peribronchial

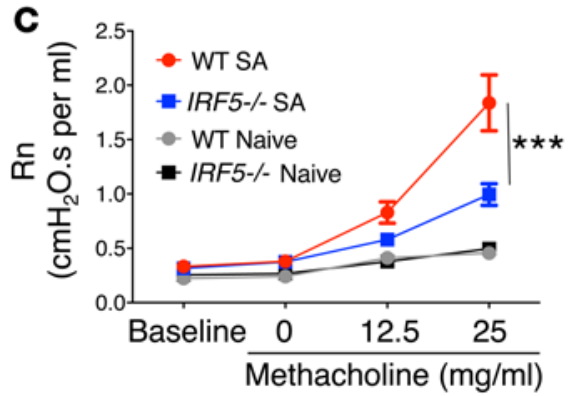

D

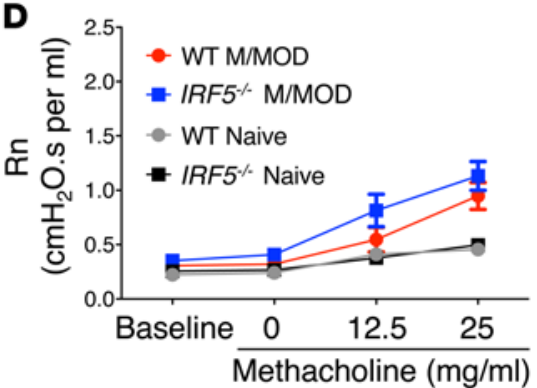

E
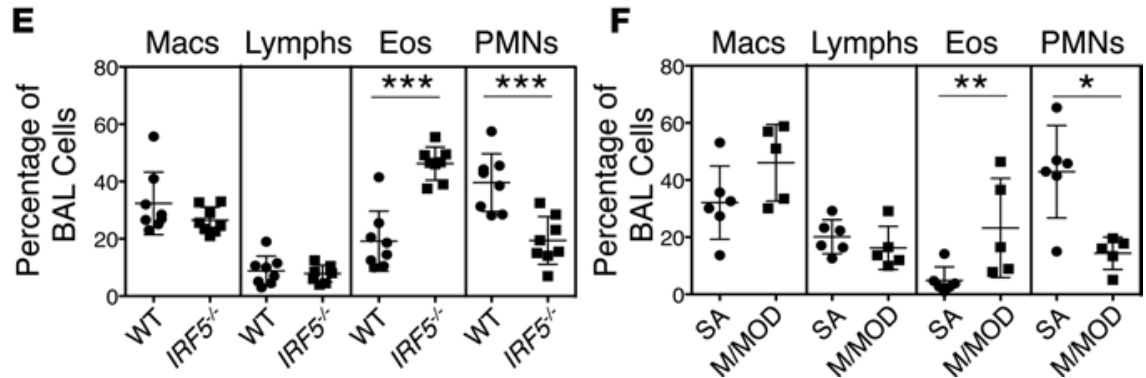

Figure 1. Upregulation of IRF5 expression in the airways of severe asthma patients and in an experimental model and its role in airway hyperresponsiveness and airway inflammation. (A, upper panel) Human bronchoalveolar lavage (BAL) cells were obtained from subjects undergoing bronchoscopy. Subjects were clinically classified as healthy controls, mild to moderate asthmatics (M/MOD), or severe asthmatics (SA). qRT-PCR was performed on total BAL cell mRNA for IRF5 expression ( $n=7 \mathrm{SA}, 6$ $M / M O D, 10$ healthy controls). (A, lower panel) Mice were sensitized with house dust mite antigen (HDM) alone (M/MOD model) or HDM + cyclic diguanosine monophosphate (c-di-GMP) (SA model). Irf5 mRNA expression was assayed by qRT-PCR in total lung homogenates. In each case, expression level relative to Hprt1 is shown. (B-F) WT or IRF5 $5^{-1-}$ mice were subjected to the model of SA or M/MOD asthma as shown. (B) Periodic acid-Schiff (PAS) stains of lung sections along with inflammation scores in the perivascular and peribronchial regions. Scale bars: $100 \mu \mathrm{m}$ ( $n=7$ per group). (C) Assessment of airway hyperresponsiveness (AHR) in mice subjected to the SA model. Shown are central airway resistance (Newtonian resistance, $\mathrm{Rn}$ ) values. (D) AHR in the M/MOD model. (E) Differential counts of BAL cells collected from WT and IRF5 ${ }^{-1-}$ mice subjected to the SA model. (F) Differential BAL cell counts similarly determined from WT mice subjected to either the SA or the M/MOD model. For all cell differentials, a total of at least 300 cells were counted and the results shown are expressed as percentage of each cell type recovered (Macs, macrophages; Lymphs, lymphocytes; Eos, eosinophils; PMNs, neutrophils). ${ }^{*} P \leq 0.05 ;{ }^{* *} P \leq 0.01 ;{ }^{* *} P \leq 0.001$ by 1 -way ANOVA with Bonferroni's multiple comparison test. $n=6-8$ mice per group (C-F). Data are the mean \pm SEM and are representative of 3 independent experiments. ns, not significant.

induces a Th2 response accompanied by low IFN- $\gamma$ expression (9) (Supplemental Figure 1; supplemental material available online with this article; https://doi.org/10.1172/jci.insight.91019DS1). As observed in humans with SA, lungs of mice sensitized with HDM + c-di-GMP for induction of the SA phenotype also showed higher Irf5 expression compared with those primed for the M/MOD model (HDM alone) or untreated animals (Figure 1A).

IRF5 deficiency impairs AHR in murine $S A$. We next utilized $I R F 5^{-1-}$ mice to determine the importance of IRF5 in promoting AHR and the high Th1 profile in the full model of SA. In order to induce a strong Th1 response, mice were sensitized with HDM in combination with c-di-GMP followed by additional challenges with HDM and a lower dose of c-di-GMP to sustain STING activation, the total time span of the model being 28 days. The general approach used was to compare the $I R F 5^{-1-}$ mice with WT animals at different levels including airway inflammation, immune priming in the lymph nodes, reactivation in the periphery, and physiologic outcome.

Histology of lung sections revealed that the overall level of inflammation was comparable between WT and $\mathrm{IRF}^{-1-}$ animals (Figure 1B). Semiquantitative, blinded scoring showed no difference in overall inflammation in either peribronchial or perivascular areas (Figure 1B). Importantly however, AHR (airway resistance, $\mathrm{Rn}$ ) in response to methacholine challenge was significantly diminished in $I R F 5^{-1-}$ mice as compared with that in WT animals (Figure 1C) in a manner similar to that in $I F N G^{-1}$ mice (9). This effect was not observed in the M/MOD model of asthma (Figure 1D) where the overall level of AHR is lower, and IFN- $\gamma$ expression is not a prominent feature, but rather where type 2 cytokines such as IL- 4 , IL-5, and IL-13 predominate (Supplemental Figure 1A) (9). Of note concerning the models themselves, c-diGMP treatment alone can induce IL-12 in APCs as we had previously shown using bone marrow-derived 
A
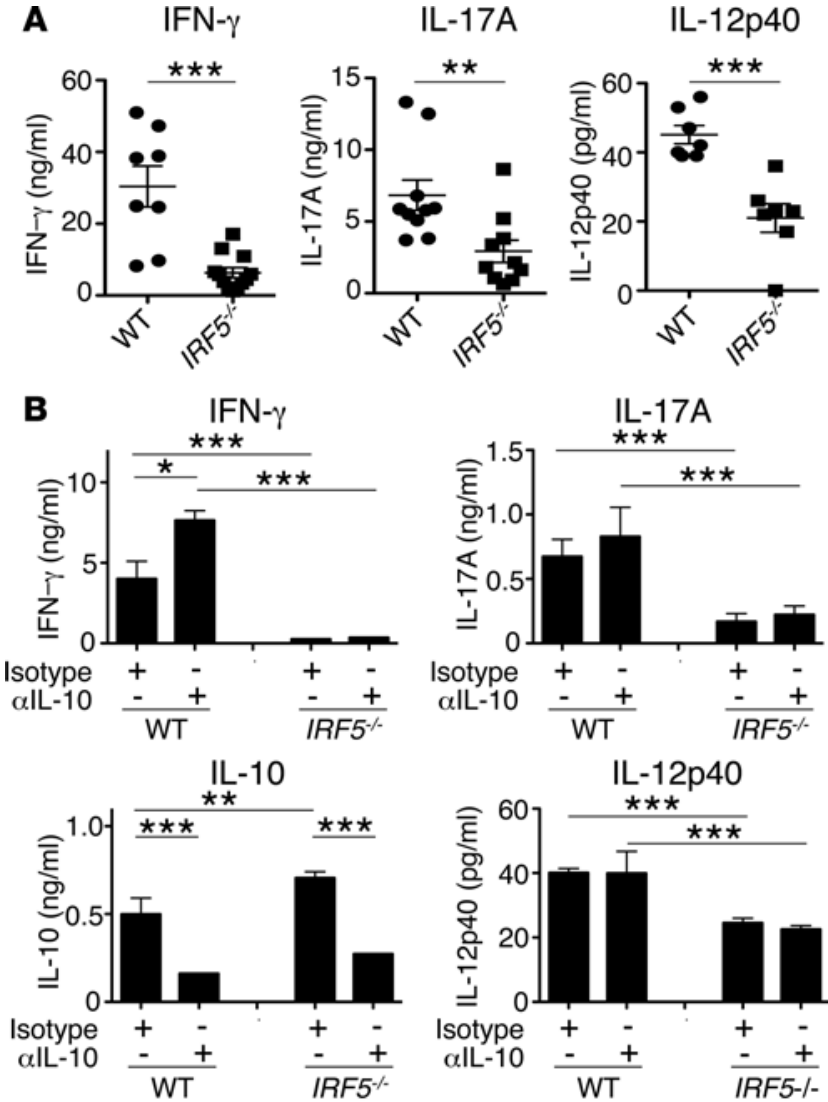

C

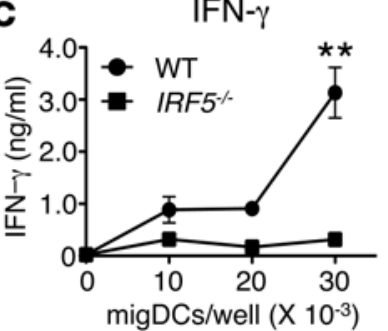

IL-5

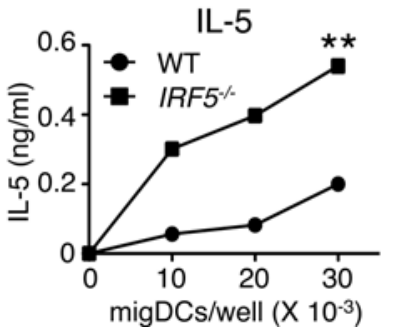

Figure 2. Decreased IFN- $\gamma$ in the lymph nodes of $I R F^{-/-}$mice in the sensitization phase of the severe asthma model. Lymph nodes were harvested 24 hours following immunization with house dust mite antigen (HDM) + cyclic diguanosine monophosphate (c-di-GMP) on days 1, 3, and 5. (A) Total lymph node cells were restimulated in vitro for 72 hours with HDM and levels of IFN- $\gamma$, IL-17A, and IL-12p40 in the culture supernatants were assessed by ELISA ( $n=8$ WT, 8-12 IRF5 ${ }^{-1-}$ mice). (B) IFN- $\gamma$, IL-17A, IL-10, and IL-12p40 production by total lymph node cells obtained from sensitized mice cultured with HDM in the presence of either neutralizing antibody against IL-10 or appropriate isotype control antibody (cells pooled from 4 to 5 mice per group and then cultured in triplicate wells). (C) Lymph node migratory DCs (migDCs) (See Figure 3 for details) and lymph node $\mathrm{CD4}{ }^{+} \mathrm{T}$ cells were both isolated by fluorescence-activated cell sorting and cultured together with HDM for 72 hours. IFN- $\gamma$, IL-17A, IL-10, and IL-12p40 production was assessed by ELISA. ${ }^{*} P \leq 0.05$; ${ }^{* *} P \leq 0.01$; ${ }^{* *} P \leq 0.001$ by Mann-Whitney $U$ test ( $\mathbf{A}$ and $\mathbf{C}$ ) or unpaired Student's $t$ test (B). Data are the mean \pm SEM and are representative of 3 independent experiments.

macrophages (BMDMs) (9), but without any accompanying antigen it does not result in $\mathrm{T}$ cell cytokine production (Supplemental Figure 1B) or any key features of experimental airway inflammation (data not shown). In marked contrast to reduced AHR in the absence of IRF5 in SA, AHR in the M/MOD model actually trended higher for the $I R F 5^{-/-}$mice, although the difference did not reach statistical significance (Figure 1D). These results suggested that a deficit in IFN- $\gamma$ production in $I R F 5^{-1-}$ mice might be responsible for decreased AHR, given that in our previous study we showed that deletion of IFN- $\gamma$ abrogates AHR in the SA model (9).

Differential cell counts of BAL cells revealed a significantly elevated percentage (Figure 1E) and number (Supplemental Figure 2) of eosinophils in $I R F 5^{-1-}$ mice compared with that in WT mice. This increased airway eosinophilia was observed in the context of total cell numbers being similar in the BAL fluid of WT and $I R F 5^{-1-}$ mice $(6.37 \pm 2.06$ versus $5.43 \pm 2.59$ million cells/mouse respectively; 8 mice/group) and in general agreement with the histologic observation of comparable overall inflammation in the 2 groups (Figure 1B). In contrast to an increase in percentage of eosinophils, a significant decrease in the percentage of neutrophils was observed in BAL cells of $I R F 5^{-/-}$mice (Figure 1E). In an earlier study of HDM-induced airway inflammation in mice, administration of anti-IL-17 neutralizing antibody decreased expression of the neutrophil chemoattractants CXCL1 and CXCL5, thereby reducing influx of neutrophils into the airways (34). Therefore, a decrease in neutrophils suggested a reduction in Th17-type cytokines, which has also been reported with IRF5 deficiency $(25,35,36)$, the association between IL-17 and neutrophils being also previously reported by us $(9,37)$. Overall, the phenotype of $I R \mathrm{F5}^{-/-}$mice subjected to the SA model resembled that of WT

mice subjected to the M/MOD model (Figure 1, E and F), which included lower AHR (compare Figure 1C $I R F 5^{-1-}$ SA to Figure 1D WT M/MOD) and predominance of eosinophils over neutrophils in BAL cells.

Lymph node DCs have reduced capacity to promote IFN- $\gamma$ production in the absence of IRF5. We next sought to determine if in fact a cytokine shift away from a type I response was occurring, specifically if IFN- $\gamma$ was reduced in $I R F 5^{--}$mice during SA, and whether it was evident at the level of immune priming in the lymph nodes and/or during the effector phase in the lung. Although the function of IRF5 in DCs has not been described heretofore in any detail, we chose to closely examine these cells in the context of IRF5 deficiency. This is because DCs, and not macrophages, are centrally involved in immune priming in the lungdraining lymph nodes following intranasal delivery of HDM, or other allergens such as ovalbumin (OVA) in combination with cholera toxin, or CpG dideoxyoligonucleotide as adjuvants (38-40). Lung DCs that 
A
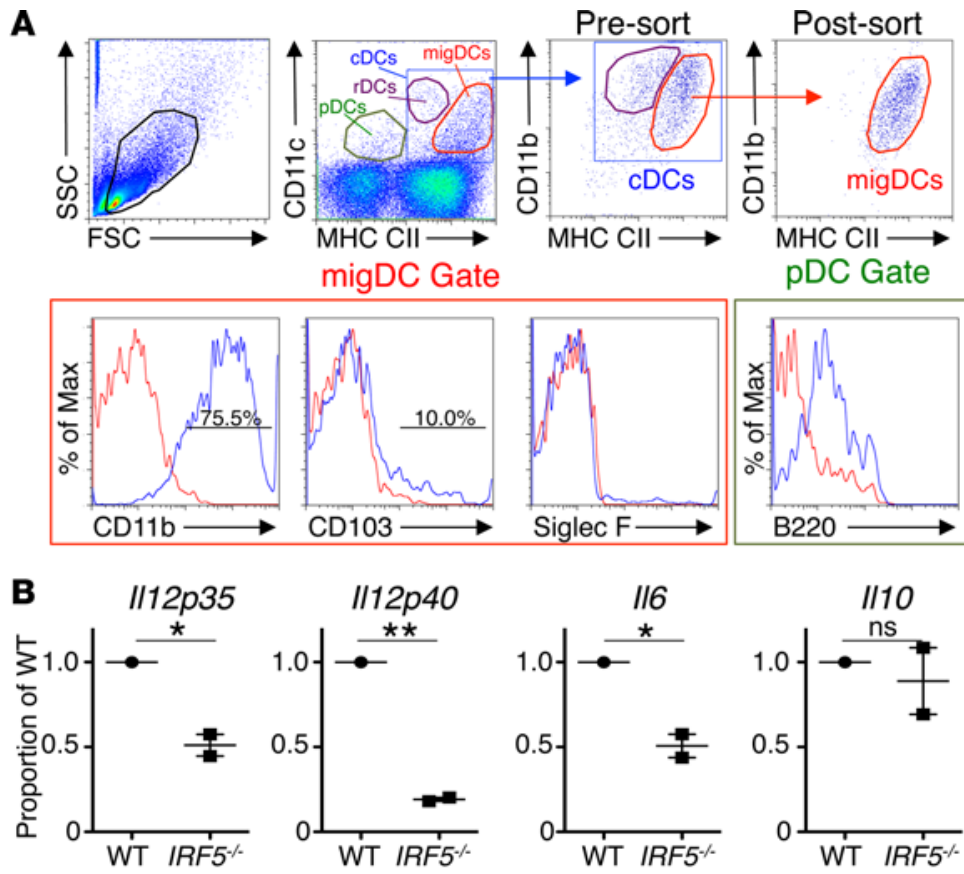

C

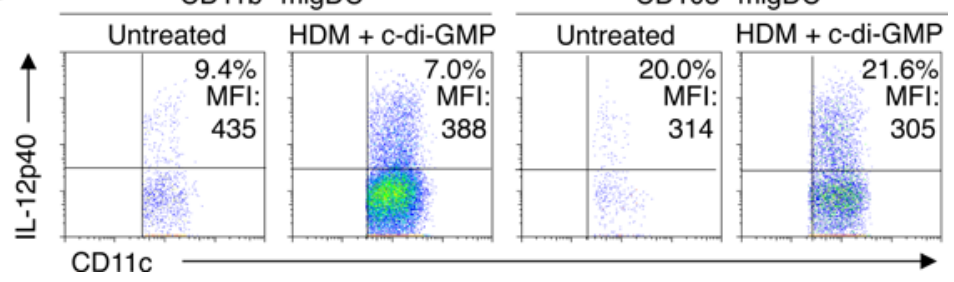

Figure 3. Migratory DCs in lymph nodes of $I R F 5^{-1-}$ mice are deficient in IL-12 and IL-6 production during the sensitization phase in model of severe asthma. Mice were sensitized as in Figure 2. (A) Migratory DCs (migDCs) with the phenotype $\mathrm{CD} 11 \mathrm{C}^{+} / \mathrm{MHC}$ class II $^{\text {hi }}$ were purified by fluorescence-activated cell sorting. migDCs contained both $\mathrm{CD}_{11 \mathrm{~b}^{+}}$and $\mathrm{CD} 103^{+}$cells at the percentages indicated. (B) qRT-PCR was performed on purified migDCs for $\|12 p 35\| 12 p 40,, \| 6$, and $\| 110$. Data are the mean \pm SEM showing proportion of expression in $I R F 5^{-/-}$mice relative to WT mice for 2 independent, combined experiments. Data are the mean \pm SEM of 7 mice per group and representative of 2 independent experiments. ${ }^{*} P \leq 0.05,{ }^{* *} P \leq 0.01$, unpaired Student's $t$ test. (C) IL-12p40-EYFP reporter mice, either untreated or sensitized with house dust mite antigen (HDM) + cyclic diguanosine monophosphate (c-di-GMP), were assessed for lymph node expression of IL-12p40 in CD11 $\mathrm{b}^{+}$and $\mathrm{CD} 103^{+}$ migDCs. Using proportions obtained by flow cytometry and total cell recovery, the number of DC subtypes found in each group was calculated on a per mouse basis and are estimated at: CD11b+ untreated, 122 cells; CD11b+ HDM + c-di-GMP-treated, 2,649 cells; $\mathrm{CD} 103^{+}$untreated, 74 cells; and $\mathrm{CD}^{-} 103^{+} \mathrm{HDM}+$ c-di-GMP-treated, 1,024 cells. Data are pooled from 3 mice per group. cDCs, conventional DCs; rDCs, resident DCs; MFI, mean fluorescence intensity; ns, not significant.

traffic to the draining lymph nodes after immunization qualitatively direct $\mathrm{T}$ helper cell differentiation via cytokines such as IL-12 and IL-6 (38-40). With regard to level of Irf5 expression, we found that expression in lung DCs is significantly higher than that in macrophages in mice treated with HDM + c-di-GMP (Supplemental Figure 3).

After priming WT and $I R F 5^{-1-}$ mice with $\mathrm{HDM}+\mathrm{c}$-diGMP (treatments on days 1, 3, and 5), the lung draining lymph nodes were harvested 24 hours later, pooled ( $4-5$ mice), and restimulated with HDM for 72 hours in vitro. At the end of the culture period, intracellular cytokine staining revealed a modest decrease in the percentage of $\mathrm{CD}^{+} \mathrm{T}$ cells capable of producing IFN- $\gamma\left(45.2 \%\right.$ versus $37.2 \%$ of total CD4 for WT and $I R F 5^{-1-}$, respectively) and IL-17 (4.3\% versus 2.3\%) (Supplemental Figure 4). The mean fluorescence intensity (MFI) of the IFN- $\gamma^{+}$cells from $I R F 5^{--}$animals was also lower, suggesting a lower cytokine/cell ratio that would collectively result in reduced levels of secreted cytokine. Indeed, there was a significant reduction in secreted IFN- $\gamma$ and IL-17 in cultures containing IRF5-deficient cells compared with those containing WT cells (Figure 2A).

IL-12p40, a component of both IL-12, the primary cytokine associated with generation of IFN- $\gamma-$ producing T cells (41-43) and of IL-23, which promotes stability of the IL-17 phenotype (44, 45), was similarly reduced (Figure 2A). IL-12p70, which is composed of the 2 subunits IL-12p35 and IL-12p40, was below the level of detection in these cultures. Although the p35 subunit of IL-12 is constitutively expressed in most cell types, the expression of the p40 subunit is inducible in hematopoietic cells such as DCs (46, 47). However, the p40 subunit of IL-12 is produced in excess relative to the p35 subunit, with the latter determining the level of secreted IL-12p70 (47). Given that the IL-12p40 levels were low at the priming stage, it is not surprising that the level of secreted IL-12p70 was below the level of detection in the lymph node cultures. We also failed to detect IL-23, presumably for similar reasons.

A key consideration, to go along with its ability to enhance expression of Th1- and Th17-promoting cytokines, is that IRF5 has also been reported to suppress expression of the immunosuppressive cytokine IL-10 (25), thus prompting us to assess the effect of blocking its function. Compared with treatment of pooled lymph node cultures in vitro with the appropriate isotype control antibody, neutralizing anti-IL-10 effectively and significantly increased IFN- $\gamma$ and IL-17 production from WT total lymph node cells, indicating that this effect was due to mitigation of the suppressive effect of IL-10 (Figure 2B). Importantly, however, in spite of IL-10 secretion being statistically significantly higher in $I R F 5^{-1-}$ cultures, its neutralization failed to enhance production of either IFN- $\gamma$ or IL-17. Whether cultures contained cells from WT and $I R F 5^{-1-}$ mice, IL-12p40 expression was unaffected by IL-10 neutralization (Figure 2B). These data showed 
A

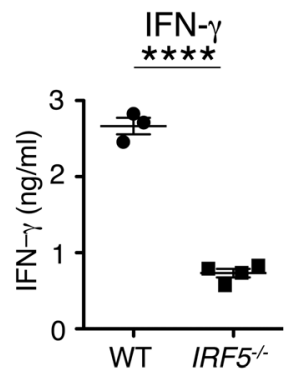

B

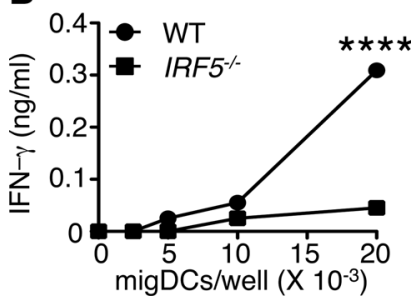

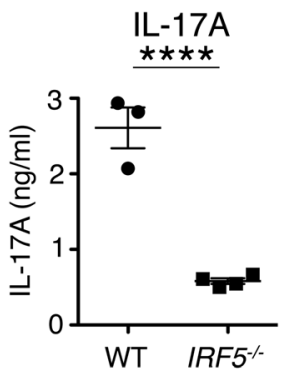

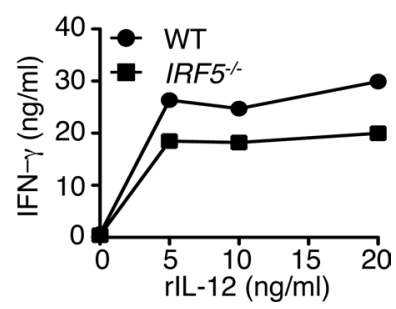

Figure 4. IRF5 ${ }^{-1-}$ DCs with deficiency in IL-12 and IL-6 production have reduced capacity to promote $\mathrm{T}$ cell IFN- $\gamma$ and IL-17A production. Lymph nodes were harvested 1 day following sensitization with OVA + cyclic diguanosine monophosphate (c-di-GMP) on days 1, 3, and 5. (A) Total lymph node cells were restimulated in vitro for 72 hours with OVA. The levels of IFN- $\gamma$ and IL-17A in the culture supernatants were assessed by ELISA ( $n=$ $3 \mathrm{WT}, 4 \mathrm{IRF5} 5^{-/-}$mice). (B) Increasing numbers of lymph node migratory DCs (migDCs) purified by cell sorting as in Figure 3 were cocultured with naive (CD62L hicD44 ${ }^{10}$ ) spleen OT-II TCR-transgenic CD4 ${ }^{+} \mathrm{T}$ cells similarly purified by cell sorting in the presence of OVA (left panel) or with increasing concentration of recombinant murine IL-12 (right panel) and secreted IFN- $\gamma$ was measured after 72 hours. Data are the mean \pm SEM of triplicate cultures of pooled cells from 3 to 5 mice per group. ${ }^{* * *} P \leq 0.0001$ by the Mann-Whitney $U$ test. Each experiment was performed twice independently.

that lower IL-12 or IFN- $\gamma$ production in the absence of IRF5 was not because of suppression by increased IL-10 levels from the DCs but that the involvement of IRF5 in the type 1 immune response was more direct.

An enhanced Th2 response was recently reported in IRF5-deficient mice subjected to an HDM-driven mild asthma model of airway inflammation $(25,48)$. In view of this finding and given that $I R F 5^{-1-}$ mice mounted lower Th1 and Th17 responses in our SA model, which would render them more prone to a Th2 response due to less cross-regulation, we also examined IL-5 and IL-13 production by lymph node T cells during priming. For these experiments, migDCs were sorted from lymph nodes of mice primed with HDM + c-di-GMP (Figure 3A and described in more detail below). The sorted DCs were cultured with sorted $\mathrm{CD}^{+} \mathrm{T}$ cells isolated from the lymph nodes of the same animals in the presence of the relevant antigen, HDM. As observed with the total lymph node cell cultures, IFN- $\gamma$ and IL-17A expression was lower when cells were derived from $I R F 5^{-/-}$mice but, as expected, levels of both IL-5 and IL-13 were higher in these cultures (Figure 2C).

Next we assessed gene expression in CD11 $\mathrm{c}^{+}$lymph node cells in more detail. Plasmacytoid DCs (pDCs) in the lung can be distinguished from conventional DCs (cDCs) based on a relatively low level of expression of MHC class II combined with B220 expression (38). A small population of pDCs was also detected in these lymph nodes (Figure 3A), but since they are not known to prime T cells for IFN- $\gamma$ production, we did not examine them further. Among cDCs, it is well established that lymph node resident DCs (rDCs) and migDCs both express CD11c but are distinguished from each other based on high MHC class II in migDCs and lower MHC class II in rDCs (49-52). During the priming phase of the SA model, lung draining lymph node migDCs were largely positive for CD11b, with only a minor population of $\mathrm{CD}_{103^{+}}$cells ( $75.5 \%$ and $10.0 \%$ of the $\mathrm{CDC}$ population, respectively), and no detectable macrophages based on dual CD11c and Siglec F staining (Figure 3A). We have found that plotting MHC class II versus

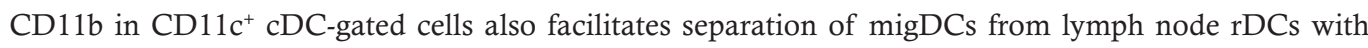
intermediate expression of MHC class II (Figure 3A). It is important to note that the DC phenotype profile shown in Figure 3A is representative of both WT and IRF5-deficient lymph node cells. Utilizing these criteria, we purified migDCs, containing both the $\mathrm{CD} 11 \mathrm{~b}^{+}$and $\mathrm{CD}_{103}{ }^{+}$subpopulations by fluorescenceactivated cell sorting. RNA was isolated from the sorted cells and quantitative reverse transcription PCR (qRT-PCR) was performed for a number of cytokines. This analysis revealed significantly decreased levels of $I l 12 p 35$, Ill2p 40, and Il6, but no difference in $I l 10$ mRNA in migDCs from IRF5 ${ }^{-1-}$ mice compared with those from WT mice (Figure 3B). The rDCs expressed several-fold lower levels of IRF5 compared with migDCs, very low Il12p35, and essentially no Il12p40 or Il6 (Supplemental Figure 5). Additionally, IRF5 has been reported to enhance expression of IL-23p19 in macrophages (25); however, Il23p19 mRNA was not detected in lymph node DCs from either WT or $I R F 5^{-1-}$ mice (data not shown).

The number of migDCs that could be obtained via cell sorting was not conducive to measuring protein from purified cells; however, we did examine IL-12 expression in the $\mathrm{CD} 11 \mathrm{~b}^{+}$and $\mathrm{CD} 103^{+}$subpopulations of migDCs using IL-12p40-EYFP reporter mice. Cells were gated based on the criteria shown in Figure 
$3 \mathrm{~A}$, and as expected, there were very few of either type of migDC in the lymph nodes of untreated animals (Figure 3C). However, even in the few migDCs identified, IL-12p40 expression was evident in both subsets. While similar data showing IL-12 expression in migratory CD103 ${ }^{+}$DCs under homeostatic conditions were reported previously (53), in our experiments we detected IL-12p40 expression in both subsets. However, the frequency of IL- $12 \mathrm{p} 40^{+} \mathrm{CD} 103^{+} \mathrm{DCs}$ was almost 3 -fold more than that of IL-12p $40^{+} \mathrm{CD} 11 \mathrm{~b}^{+}$ DCs (Figure 3C). Upon sensitization with HDM + c-di-GMP, we detected a substantial increase in the numbers of both $\mathrm{CD}_{11 b^{+}}$and $\mathrm{CD}_{103^{+}}$migDCs in the lymph nodes, which maintained similar frequencies of IL-12p $40^{+}$cells as observed under homeostatic conditions ( $7 \%$ versus $21.6 \%$, respectively) (Figure $3 \mathrm{C}$ ). This increase in IL-12p $40^{+}$DCs upon priming of the mice for SA was in accordance with increased Irf5 expression detected in the lungs of immunized mice (Figure 1A). Our data suggest that both $\mathrm{CD}_{103^{+}} \mathrm{DCs}$ and $\mathrm{CD} 11 \mathrm{~b}^{+} \mathrm{DCs}$ contribute to Th1 priming in our SA model, although we have not formally ruled out the possibility that the function of one or the other is differentially altered in the absence of IRF5, which will be addressed in future studies.

Given the difference in IL-12 expression in migDCs between WT and $I R F 5^{-1-}$ mice, their function in promoting $\mathrm{T}$ cell priming and differentiation was examined. This series of experiments was performed using the OVA system because of availability of OVA-specific TCR-transgenic mice, OT-II. Mice were treated in a manner identical to priming in the SA model with c-di-GMP, but with OVA substituted for HDM. Use of OVA as an antigen also resulted in decreased IFN- $\gamma$ and IL-17A in total lymph node cell cultures established from immunized $\mathrm{IRF5}^{-1-}$ mice (Figure 4A), demonstrating that IRF5-mediated cytokine modulation is not specific to HDM. Lymph node migDCs were then purified as described above, and cocultured in vitro with OVA, and naive $\left(C D 62 \mathrm{~L}^{\mathrm{hi}} \mathrm{CD} 44^{\mathrm{lo}}\right)$ OT-II TCR-transgenic spleen T cells, which were also purified by cell sorting. IFN- $\gamma$ was detected in culture supernatants at 72 hours of incubation, albeit at expectedly low levels at this early time in $\mathrm{T}$ cell differentiation, and expression

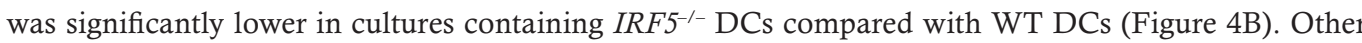
cytokines were below the level of detection at this early time point. These data suggested that decreased IL-12 production in IRF5-deficient mice is directly and mechanistically linked to deficiency in IFN- $\gamma-$ promoting capacity during priming in the lymph nodes. To further support this notion, recombinant IL-12 (rIL-12) was added to cultures containing OT-II T cells and migDCs from WT or $I R F 5^{-1-}$ mice. As shown in Figure 4B, rIL-12 supplementation was able to support a high level of IFN- $\gamma$ production from the T cells whether DCs were used from WT or IRF5-deficient mice, showing that IL-12 deficiency is the limiting factor in the absence of IRF5 (Figure 4B). Since Il6 gene expression is also severely impaired in IRF5-deficient migDCs (Figure 3B), we expect that IL-6 supplementation in the cocultures will similarly boost IL-17 production from the T cells.

Significantly decreased IFN- $\gamma$ and IL-17A but increased IL-5 in lung tissue in IRF5 ${ }^{-1-}$ mice. In order to determine if the $I R F 5^{-1-}$ mice display a deficit in IFN- $\gamma$ in the lungs when subjected to the SA model, the full 28-day model was run and total lung extracts were prepared for RNA and protein estimation. qRT-PCR revealed the expected reduction of Ifng mRNA in $I R F 5^{-1-}$ mice relative to that in their WT counterparts (Figure 5A). Whole-lung $I l 17$ mRNA levels were not appreciably different between the mice, but mRNA expression for the type 2 cytokines IL- 4 , IL-5, and IL-13 was increased in $I R F 5^{-1-}$ mice relative to that in WT animals, although among these only the difference in $I l 5$ levels reached statistical significance (Figure 5A). It is possible that $I l 17 \mathrm{mRNA}$ expressed by other cell types such as $\gamma \delta \mathrm{T}$ cells, known to be a rich source of IL-17, contribute to $I l 17$ mRNA expression in the absence of IRF5-driven IL-6. Indeed, lack of IL-6 was found to have a drastic effect on IL-17 expression in $\mathrm{CD}^{+} \alpha \beta \mathrm{T}$ cells but had a minimal effect on IL-17 production from $\gamma \delta \mathrm{T}$ cells (54). These cells are not as numerous as $\mathrm{CD} 4^{+} \mathrm{T}$ cells in most inflammatory settings but may express the mRNA at a high level constitutively as suggested from IL-17 production by these cells even in naive mice (55). With regard to the Th2 cytokines, as was the case in the lymph nodes, the trend towards higher type 2 cytokines in $I R F 5^{-1-}$ mice is most likely due to immune cross-regulation since no direct transcriptional effect of IRF5 on Th2 differentiation has been described to our knowledge $(25,48)$. We also assayed cytokine protein levels in the lungs of WT and $I R F 5^{-1-}$ mice in the presence or absence (control) of the CS, dexamethasone (Dex). At the protein level, secreted IFN- $\gamma$ and IL-17A in the

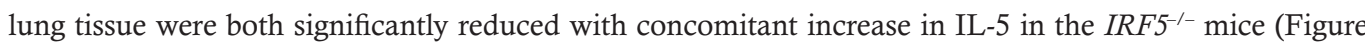
5B). Roughly parallel to the mRNA, IL-13 trended upward in the $I R F 5^{-/-}$animals but IL-4 protein was actually reduced, although not to a statistically significant degree. Treatment with Dex suppressed the increased protein levels of both IL-5 and IL-13 in $\mathrm{IRF5}^{-1-}$ mice (Figure 5B). At both mRNA and protein levels, IL-10 

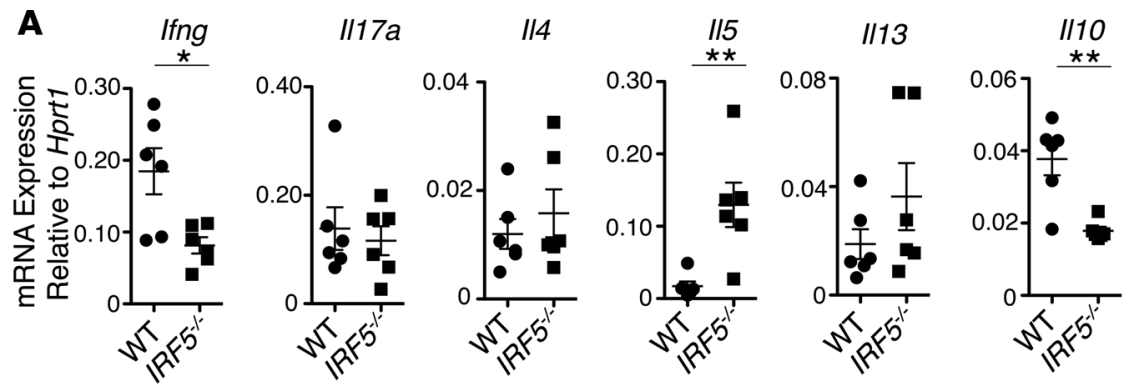

IL-4
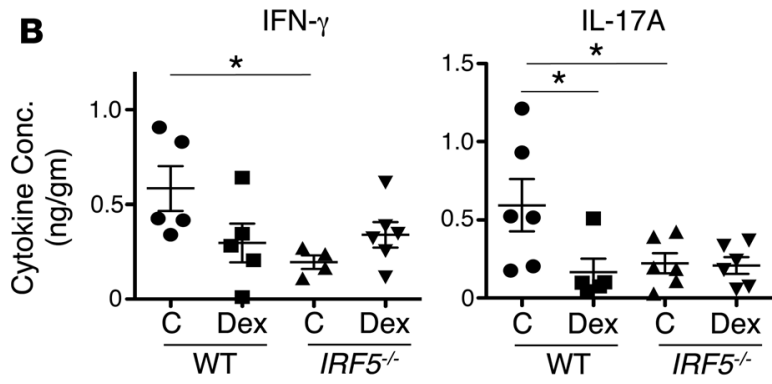

IL-13

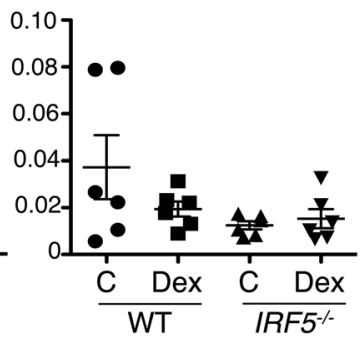

$\mathrm{IL}-10$
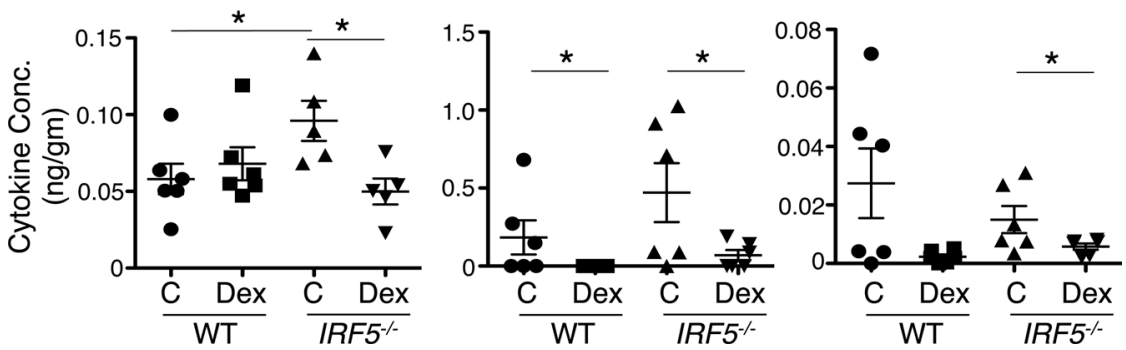

Figure 5. Reduced IFN- $\gamma$ and IL-17 but increased IL-5 and IL-13 levels in lungs of IRF5 ${ }^{-/-}$mice subjected to the severe asthma model. WT or IRF5 $5^{-1-}$ mice were subjected to the full 28-day model of severe asthma with or without (control [C]) treatment with dexamethasone (Dex). (A) Cytokine mRNA levels in whole-lung homogenates measured by qRT-PCR. (B) Cytokine protein levels in lung tissue homogenates. ( $\mathbf{A}$ and $\mathbf{B} ; n=4-6$ mice per group). Data are the mean \pm SEM and each experiment was performed at least twice. ${ }^{*} P \leq 0.05,{ }^{*} P$ $\leq 0.01$ using the Mann-Whitney $U$ test.

was lower in the lungs of $I R F 5^{-/-}$mice. However, more work is needed to determine the role of IRF5 in Il10 gene expression in individual cell types, especially in migDC versus lung rDC subsets and in lung macrophages. Il9 mRNA expression was not detected in our model.

The cytokine expression profile in $\mathrm{IRF5}^{-{ }^{-}}$and WT mice likely explained the concomitant lower AHR (Figure 1C) due to decreased IFN- $\gamma$. Also, the higher airway eosinophilia in the absence of IRF5 (Figure $1 \mathrm{E}$ ) could be explained by the increase in $I l 5$ gene expression that was observed in these animals (Figure 5A). While a lower IFN- $\gamma$ response in the absence of IRF5 would be beneficial in SA, the resulting enhanced $\mathrm{Th} 2$ response would actually be detrimental. In other words, benefits achieved with improvement in airway function by inhibiting IRF5 would be offset by its sequelae, one of which is increased airway eosinophilia. These data show that IRF5 has an important effect on the balance between effector cytokines in the context of the complex immune response in SA and targeting IRF5 alone may not be a viable standalone approach to therapeutic intervention in severe disease.

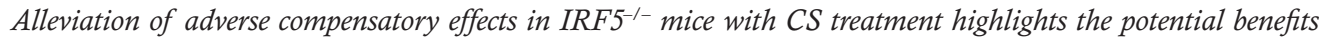
of combined therapy. The most critical issue regarding SA in humans is that the disease condition is often poorly controlled, in spite of the fact that individuals almost invariably are maintained on high-dose CS (4), leading to profoundly adverse quality of life issues (5). The data presented thus far show that inhibition of one arm of the immune response could have beneficial effects in vivo; however, that same intervention also might explain the undesirable reciprocal effect of augmenting type 2 cytokines and the eosinophilic response in the airways. Therefore, we evaluated the combined effects of IRF5 deficiency and CS treatment as an approach to simultaneously address the unwanted effects of the diverse immune pathways leading to the complex immune phenotype in SA. The rationale was that since the immune response in IRF5-deficient animals subjected to the SA model seems to mirror the response in M/MOD asthma model (Figure 1), it might be amenable to CS treatment, as observed in humans with M/MOD asthma (1-3).

Treatment of WT mice with dexamethasone (Dex) slightly reduced IFN- $\gamma$ (not statistically significant) and had a more marked effect on IL-17A protein level in WT mice (Figure 5B). No inhibitory effect on IL-5 was 
A

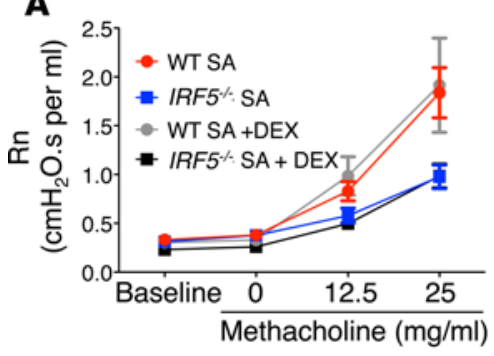

C

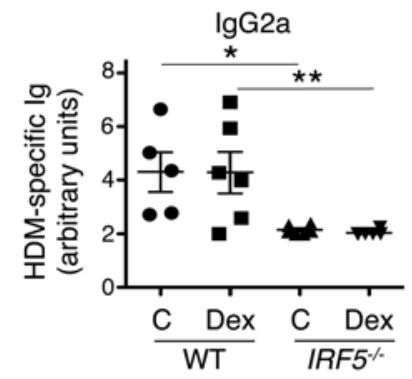

D

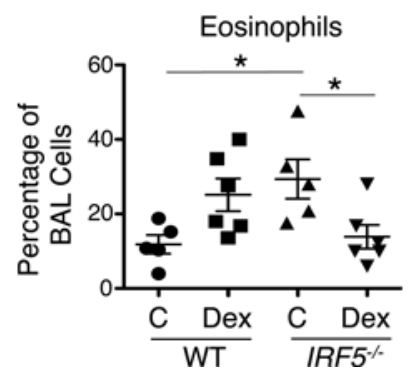

B

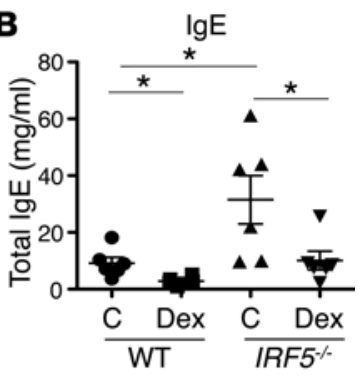

$\lg \mathrm{G} 1$
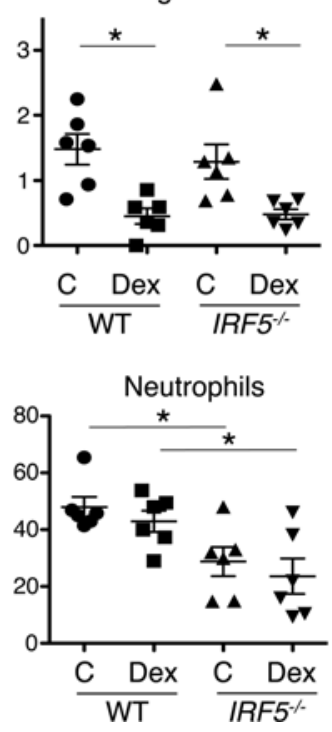

Figure 6. Combination of IRF5 deficiency and corticosteroid treatment alleviates multiple features of severe asthma. WT or IRF5 $5^{-1-}$ mice were subjected to the full 28-day model of severe asthma (SA) with or without (control [C]) treatment with (Dex). (A) Central airway resistance (Newtonian resistance, $\mathrm{Rn}$ ) measurements are shown (6-8 mice were used per group). (B) Levels of total serum IgE and (C) house dust mite antigen-specific (HDMspecific) IgG2a and IgG1. (D) Percentage of eosinophils and neutrophils in bronchoalveolar lavage (BAL) cells. $n=5-6$ mice per group (B-D). Data are the mean \pm SEM and each experiment was performed at least twice independently. $P \leq 0.05,{ }^{* *} P \leq 0.01$ using the Mann-Whitney $U$ test.

noted. In IRF5 $5^{-1-}$ mice, while IFN- $\gamma$ and IL-17A protein levels were both lower with no change upon Dex treatment, the increased levels of IL-5 and IL-13 protein in $I R F 5^{--}$mice were suppressed by Dex treatment (Figure 5B). Interestingly, for reasons that are unclear at this time, IL-13 but not IL-5 protein level in the lung was reduced upon Dex treatment of WT mice. The level of IL-4 remained similar regardless of Dex treatment, while that of IL-10 was somewhat reduced by Dex (Figure 5B).

We next assessed the effect of Dex on AHR in the 2 groups of mice at the end of the full SA model. As shown in Figure 1, the degree of AHR in $\mathrm{IRF5}^{-1-}$ mice was significantly lower and comparable to low AHR in WT animals subjected to the M/MOD model (compare Figure 1, C and D, and Figure 6A). Dex did not further reduce AHR in either WT or $I R F 5^{-1-}$ animals (Figure 6A). As observed previously, the inability of Dex to further reduce the low level of AHR in the M/ MOD model may be due to a low level of IFN- $\gamma$ in the lungs of these mice, since in a model of asthma driven by Th2 cytokines only, AHR is completely blunted by Dex (9). We also measured serum Ig levels in the mice with or without Dex treatment. Since the high antigenspecific IgG levels preclude assay of the relatively low antigen-specific IgE levels, total IgE and HDM-specific IgG2a and IgG1 levels were measured. The IgE level was low in WT mice subjected to the SA model, which was further reduced by Dex (Figure 6B). The level was higher in the $I R F^{-/-}$mice, which was suppressed by Dex (Figure 6B). The profile of IgE in the different groups mirrored the level of IL-13 in the lungs of the mice, this cytokine being a key driver of IgE levels in both humans and mice (56-58). HDM-specific IgG2a, the generation of this isotype being driven by IFN- $\gamma$ (59), was significantly inhibited in $I R \mathrm{F5}^{--}$mice, with no further reduction by Dex (Figure 6C). The level of HDM-specific IgG1 was reduced in both WT and $I R F 5^{-1-}$ mice by Dex and largely paralleled IL-4 levels in the mice, which mediates switching to IgG1 (60).

At the level of airway inflammation, in line with the unaltered IL-5 level in the lungs of Dex-treated WT mice (Figure 5B), no reduction in eosinophils was observed (Figure 6D). This agreement between lung IL-5 levels and eosinophils was also evident in the $I R F 5^{-/-}$mice, which showed higher IL-5 (Figure 5B) and higher eosinophils (Figure 6D). However, these mice showed lower lung IL-5 and eosinophils upon Dex treatment (Figure 5B and Figure 6D). Despite lowering of IL-17 by Dex, BAL neutrophils did not decrease appreciably in WT mice (although there was a trend). However, the BAL neutrophils were lower in $\mathrm{IRF5}^{-/-}$mice, with or without Dex, consistent with lower IL-17 levels in the lungs of these mice (Figure 6D). Collectively, these results indicate that in the SA model, IRF5 has a major role in promoting both IFN- $\gamma$ and IL-17 responses in $\mathrm{CD} 4^{+} \mathrm{T}$ cells. Also, our data show that lack of IRF5 can promote a Th2 response even in mice immunized to develop high Th1 and Th17 responses. Importantly, the heightened type 2 response and airway eosinophilia in the $I R F 5^{-1-}$ mice can be attenuated with CS. Overall, our findings suggest that it may be possible to target specific immune processes with combined therapeutic approaches in SA, where treatment options currently are limited.

\section{Discussion}

We recently reported that the BAL cells of more than $50 \%$ of severe asthmatics produce high levels of IFN- $\gamma$, despite treatment of these subjects with high-dose CS (9). Using a model of SA developed by us that also displayed a high level of IFN- $\gamma$ T cells that was marginally inhibited by CS, we showed that IFN- $\gamma$ 
is critical for the development of AHR (9). This type of cytokine profile is unlike the more commonly encountered Th2 response in the airways of subjects with milder allergic asthma (9). Given that significantly reduced lung function and increased AHR along with high IFN- $\gamma$ expression are cardinal features in a significant proportion of severe asthmatics, we evaluated the role of IRF5 as a potential upstream regulator of AHR via IFN- $\gamma$ in our model system. This is because IRF5 expression in human macrophages was shown to impart IFN- $\gamma$-inducing capacity to cocultured $\mathrm{CD}^{+} \mathrm{T}$ cells (25). In this study, we focused on the role of IRF5 in DCs in inducing expression of IL-12, which is the key driver of Th1 differentiation. DCs, and not macrophages, typically migrate to lung-draining lymph nodes to prime $\mathrm{T}$ cells except during infections by specific pathogens such as pneumococci (61). Also, lung macrophages and DCs can express distinct cytokine and chemokine genes in response to the same pathogen, Mycobacterium tuberculosis (62), and thus a role for IRF5 in driving IL-12 production in BMDMs or monocyte-derived macrophages does not assure a similar role in lung DCs. More importantly, since the role of IRF5 in priming and reactivation of a Th1 response in vivo has not been examined before, we investigated whether this molecule determines a high level of IFN- $\gamma$ production from T cells in a model of SA. We demonstrate that a deficiency in IRF5 drastically impairs expression of both the subunits of IL-12 as well as of IL- 6 that drives Th17 differentiation, in lung migDCs. The deficit in IL-12 and IL-6 production in IRF5 $5^{-1-}$ DCs was found to have a direct functional impact on Th1 and Th17 differentiation from naive $\mathrm{CD} 4^{+} \mathrm{T}$ cell precursors in vitro. Reduced IFN- $\gamma$ and IL-17 levels in vivo were associated with markedly decreased AHR and airway neutrophilia. These data suggest that IRF5 expression level in asthmatics has predictive value for disease severity in terms of pulmonary function and reinforces the notion that this molecule is a viable candidate to target for therapeutic intervention.

Based on the cycle threshold $(\mathrm{Ct})$ values in our qRT-PCR data, there is some basal level of Irf5 expression in both humans and mice. In fact, low levels of constitutive nuclear IRF5 have been previously described (63), which may function as a homodimer in association with coactivators to promote basal transcription of target genes. This may account for constitutive IL-12 expression in the small number of migDCs detected in the draining lymph nodes of the IL-12p40-EYFP reporter mice. IL-12 expression was detected in both $\mathrm{CD}_{11 b^{+}}$and $\mathrm{CD} 103^{+}$migDCs, albeit at a higher frequency in the latter. It is interesting to note that even though we found that $\mathrm{CD} 103^{+}$DCs comprise only $10 \%$ of migDCs in the lymph nodes compared with $75 \%$ in the case of CD $11 b^{+}$DCs, given that 3 times more of the CD $103^{+}$DCs express IL- 12 , the absolute number of CD103 $\mathrm{IL}-12^{+} \mathrm{DCs}$ is a little less than half that of CD11 $\mathrm{b}^{+} \mathrm{IL}-12^{+} \mathrm{DCs}$. A previous study also reported constitutive IL-12 expression in migratory CD $103^{+} \mathrm{DCs}$ in lung-draining lymph nodes, and these cells were found to suppress an anti-helminth type 2 response, promoting chronicity of infection (53). Also, we showed that in immune tolerance induced by a low dose of inhaled antigen, the CD103+ DCs upregulate expression of the key enzyme for retinoic acid (RA) production, retinaldehyde dehydrogenase (Aldh1a2), RA being a potent cofactor for FOXP3 expression in Tregs (64). We showed that under conditions of tolerance, CD103 ${ }^{+}$DCs are superior to all other DC subsets and also alveolar macrophages in inducing FOXP3 expression in $\mathrm{CD}^{+} \mathrm{T}$ cells (64). Although constitutive IL-12 expression by the CD103 DCs may affect the anti-helminthic response because of suppression of Th2 development, this cross-regulatory effect may actually help to prevent the development of a Th2 response against aeroallergens that drives mild allergic asthma. However, a heightened persistent Th1 response is deleterious and promotes more severe asthma that is poorly controlled by CS. Thus, it appears that CD $103^{+}$DCs are functionally the most dynamic, being able to alter their gene expression profile to promote either Treg or Th1 development.

Higher IRF5 mRNA expression in SA may be due to single nucleotide polymorphisms (SNPs) in the IRF5 gene that are known to increase IRF5 transcript levels or due to increased transcription induced by pathogen-induced signaling mechanisms or pathogen-associated products. Several SNPs in the IRF5 gene that cause an increase in steady-state IRF5 transcript levels have been associated with Th1-mediated diseases like systemic lupus erythematosus and rheumatoid arthritis (35). Viral infections have been shown to promote IRF5 mRNA levels, and while the 5' UTR of the IRF5 gene harbors binding sites for multiple transcription factors, there is limited knowledge about mechanisms underlying IRF5 gene expression (35). We previously showed that lipid-mediated transfection of c-di-GMP into BMDMs causes a robust increase in expression of both subunits of the $I l 12$ gene in a STAT1-dependent fashion, which suggests that c-diGMP induces Irf5 transcription or IRF5 activation or both (9). However, given that we detect more Irf5 transcripts in the lungs of mice subjected to the SA model, this bacterial product most likely augments both Irf5 transcription via STAT1 as well as its activation. Indeed, activation of the kinase TBK1 by c-di-GMP has 
been linked to activation of multiple IRF family members including IRF3, IRF5, and IRF7 (29). Similarly, LPS has been shown to promote activation of IRF5 and NF-kB, and the heterodimer of IRF5 and RelA can bind to the promoter of multiple inflammation-associated genes (24). Taken together, it appears that increased transcription and activation of IRF5 in concert with other transcription factors such as NF- $\mathrm{BB}$ causes an increase in influx of IL-12-expressing $\mathrm{CD} 11 \mathrm{~b}^{+}$and $\mathrm{CD} 103^{+} \mathrm{DCs}$ in the lymph nodes, although the percentage of migDCs expressing IL-12 in immunized mice remains similar to that in naive mice. A net increase in the number of IL-12-, and by extension, of IL-6-expressing DCs in the lymph nodes helps to drive Th1 and Th17 differentiation of naive $\mathrm{CD}^{+} \mathrm{T}$ cells. The heightened Th1/Th17 phenotype appears to be maintained when the effector $\mathrm{T}$ cells emigrate to the periphery. The molecular mechanisms that guide basal versus induced transcription of Irf5 may, however, be distinct.

It is important to note that some severe asthmatics present with a high eosinophilic response, and maintain eosinophilia despite being on treatment with a high dose of CS. It will be interesting to determine whether IRF4, which belongs to the same family of transcription factors, and previously shown to promote Th2 development in a mild asthma model (65), is responsible for the type 2 bias in some of these subjects. Increased IRF4 expression associated with a Th2 response was noted in CD11b+ DCs in the previous study (65). Since in the present study IRF5 expression was detected at a higher frequency in CD $103^{+}$DCs compared with $C D 11 b^{+}$DCs, it is possible that in SA with a high type 2 response, IRF4 dominates over IRF5 even in $\mathrm{CD} 103^{+} \mathrm{DCs}$. In fact, IRF4 is known to competitively inhibit IRF5 binding to MyD88 downstream of TLR signaling independent of the STING pathway, such that the interaction and balance between these 2 molecules could determine type 2-dominated versus type 1-dominated immune phenotypes in SA (66).

Whereas investigations are ongoing in our laboratory to determine the molecular basis for CS insensitivity in all severe asthmatics, our current study suggests that in patients displaying a Th1/Th17hi immune profile, targeting IRF5 with small-molecule inhibitors in combination with CS will achieve the best results. Since this combined approach has the potential to inhibit Th1, Th2, and Th17 immune responses, severe asthmatics should experience improvements in lung function, along with suppression of airway eosinophilia and neutrophilia, and reduced serum Ig levels. It is also possible that dampening the Th1/Th17 response by inhibiting IRF5 may induce a steroid-sparing effect. It has been suggested that enhancing IRF5 function in milder asthma might be therapeutically beneficial due to inhibition of Th2 response by enhanced Th1 response (48). However, in aggregate, our previous and present studies highlight the deleterious consequence of persistent Th1 response that induces more severe disease that is poorly controlled by CS. The findings in the present study suggest that inhibition of IRF5 alone in SA could potentially result in a M/ MOD-type condition in the long run given the observed increase in both $\operatorname{IgE}$ and airway eosinophils in the absence of IRF5. Thus, a multifaceted approach to therapy is important in SA where the immune-related pathology is complex and highly heterogeneous in nature $(2,3)$. In conclusion, a critical evaluation of the immune response in the airways of asthmatics along with expression levels of regulators of the immune response such as IRF5 would constitute a more personalized approach to treating asthma, which is increasingly being advocated and practiced.

\section{Methods}

Human BAL cells. Human subjects were recruited as part of the Severe Asthma Research Program (SARP). Bronchoscopy to obtain BAL cells was performed according to previously published guidelines and procedures (67). Each subject was categorized as severe asthmatic, M/MOD asthmatic, or healthy control based upon the latest European Respiratory Society/American Thoracic Society (ERS/ATS) criteria (4). Total BAL cell mRNA was isolated (RNeasy Kit; QIAGEN) from samples that had been immediately placed in RLT buffer (QIAGEN) and qRT-PCR was performed as described below using a human primer and probe set (Hs00158114_ml; Thermo Fisher Scientific).

Mice. IRF5-deficient mice originally derived by Tak Mak at the University of Toronto (Toronto, Ontario, Canada) and backcrossed 8 generations onto the C57BL/6J background were provided to us by Ian Rifkin at Boston University (Boston, Massachusetts, USA) after further backcrossing of another 15 generations following removal of an original parallel mutation in DOCK2 (68). The DOCK2 mutation had introduced potentially confounded results obtained using the $I R F 5^{-1-}$ mouse strain to study B cell development since both IRF5 and DOCK2 were reported to be potentially involved. (69). OT-II (Thy 1.1) mice were a gift of Lauren Cohn (Yale University, New Haven, Connecticut, USA). All mice were bred in-house at the University of Pittsburgh. WT C57BL/6J controls and IL-12p40-EYFP reporter mice $\left(B 6.129-I l 12 b^{\text {tm/Lky }} / J\right.$; 
catalog 006412) were purchased from The Jackson Laboratory. All animals were housed under germ-free conditions and were used for experiments between 8 and 10 weeks of age.

Models of SA and M/MOD asthma. These models have been described and evaluated in detail by us previously (9). Briefly, the SA model consists of a sensitization phase where $25 \mu \mathrm{g}$ of HDM antigen (Greer Laboratories; lot selected for low endotoxin) is administered intranasally to lightly anesthetized mice on days 1,3 , and 5 along with $5 \mu$ g of the bacterial product c-di-GMP (Biolog Inc.). Following a rest period of 5 days, the challenge phase consisted of 3 sets of identical treatments each separated by 4 days of rest as follows: day 1, $25 \mu \mathrm{g} \mathrm{HDM}+5 \mu \mathrm{g}$ c-di-GMP; days 2 and 3, $25 \mu \mathrm{g}$ HDM alone. Experiments aimed at analysis of the sensitization phase were performed on day 6 and those examining the full model on day 28. The M/MOD asthma model was identical to the above except that no c-di-GMP was used. For experiments involving in vitro culture of APCs with OT-II TCR-transgenic CD4 $4^{+} \mathrm{T}$ cells, $100 \mu \mathrm{g}$ of OVA low endotoxin; Sigma-Aldrich) was used in place of HDM. In experiments involving steroid treatment, Dex (Baxter Inc.) was administered intraperitoneally at $4 \mathrm{mg} / \mathrm{kg}$ beginning on the first day of challenge. These treatments were repeated every 3 days throughout the duration of the challenge phase of the model.

Pulmonary function testing for assessing AHR. We have described assessment of AHR in mice in detail previously (9). Briefly, mice were anesthetized and subjected to the forced oscillation technique for measuring AHR using a Flexivent PFT apparatus (SCIREQ). Measurements of lung function were made following perturbation with increasing doses of methacholine.

Differential BAL cell counting and lung histology. BAL was performed on anesthetized mice by infusion and recovery of $1 \mathrm{ml}$ sterile PBS. Approximately $1 \times 10^{5}$ BAL fluid cells were placed onto glass slides by cytospin. Following air drying these slides were stained with Giemsa. Macrophages, lymphocytes, eosinophils, and neutrophils were enumerated out a total of approximately 300 cells.

For histology, lungs fixed in SafeFix II (Thermo Fisher Scientific) were embedded in paraffin, sectioned, and stained with periodic acid-Schiff (PAS) reagent. An inflammation score on a scale of 0 to 4 was assigned to the perivascular and peribronchial areas of multiple fields per slide in blinded fashion based on previously published methodology (70-73). Briefly, a score of zero represented complete lack of inflammation and a designation of 4 was assigned to areas where inflammatory cells were present at a depth of greater than 3 cells completely surrounding an airway or blood vessel. Approximately $20-50$ consecutive $\times 400$ fields were examined in each slide. The sum of the inflammatory scores was divided by the total number of airways or blood vessels to arrive at the final composite value for each animal. Approximately 8 mice per condition were scored in this manner. BAL cell differentials and lung histology slides were all read, and photomicroscopy performed, using a Zeiss Axiophot microscope mounted with a Zeiss AxioCam HRc camera.

Flow cytometry. Directly labeled antibodies were used to stain surface markers in single-cell suspensions in a volume of $100 \mu \mathrm{FACS}$ buffer (PBS $+2 \% \mathrm{FCS}$ ) for 20 minutes at $0^{\circ} \mathrm{C}$. For these experiments, antibodies against the following molecules were used: CD4-PerCP Cy5.5 (clone RM4-5; catalog 550954; 1:200), CD11c-APC (clone HL3; catalog 550261; 1:200), CD11b-PE (clone M1/70; catalog 553311; 1:200), CD103-PE (clone M290; catalog 557495; 1:200), SiglecF-PE CF594 (clone E50-2440; catalog 552126; 1:200), CD45R/B220-PE (clone RA3-6B2 catalog 553090; 1:200) all from BD Biosciences, and MHC class II-FITC (clone NIMR-4; catalog 1895-02; 1:200) from SouthernBiotech. In all cases, appropriate isotype control antibodies were used in parallel. Data acquisition was performed on a FACSAria flow cytometer (BD Immunocytometry Systems). Final analysis was performed using FlowJo software (Tree Star).

Cell isolation and culture. Our cell isolation methods have been described in detail previously (9, 38, 39, 64). Briefly, single-cell suspensions were first prepared from the lung-draining lymph nodes. Lymph node migDCs were sorted from the single-cell suspensions based upon the phenotype CD $11 \mathrm{c}^{+} / \mathrm{MHC}$ class $\mathrm{II}^{\mathrm{hi}}$ / $\mathrm{CD}_{11 b^{+}}(49)$ using the FACSAria cell sorter. For certain experiments, lymph node CD4 ${ }^{+} \mathrm{T}$ cells were simultaneously sorted from the same single-cell suspension. Naive CD4 ${ }^{+}$TCR-transgenic T cells were isolated from spleens of OT-II mice using a T cell isolation kit (Miltenyi Biotec), followed by cell sorting for the phenotype CD4+CD62L+CD44 ${ }^{-}$. CD62L-PE (clone MEL-14; catalog 553151; 1:200) and CD44-FITC (clone IM7; catalog 553133; 1:200) antibodies were both from BD Biosciences.

For in vitro restimulation, total lymph node cells were plated at $1 \times 10^{6}$ cells $/ \mathrm{ml}$ in complete cell culture medium: RPMI 1640 (Gibco) supplemented with 10\% heat-inactivated FBS (Gemini), $100 \mathrm{U} / \mathrm{ml}$ penicillin, $100 \mu \mathrm{g} / \mathrm{ml}$ streptomycin sulfate (Gibco), $1 \mathrm{mM}$ sodium pyruvate (Gibco), and $50 \mu \mathrm{M}$ 2-mercaptoethanol (Sigma-Aldrich). Depending upon the in vivo model being used, appropriate antigen, HDM $(100 \mu \mathrm{g} / \mathrm{ml})$ or OVA $(100 \mu \mathrm{g} / \mathrm{ml})$ was added. In certain experiments, $10 \mu \mathrm{g} / \mathrm{ml}$ no-azide/low-endotoxin 
anti-IL-10 neutralizing antibody (JES5-16E3; catalog 554463) or appropriate control antibody (A95-1; catalog 553985), both from BD Biosciences, was added for the duration of the culture incubation. Cultures were incubated for 72 hours and then soluble cytokine expression was assessed by ELISA. Additional in vitro restimulation experiments were performed following in vivo sensitization by coculturing purified lymph node $\mathrm{CD} 4^{+} \mathrm{T}$ cells $\left(5 \times 10^{5}\right.$ cells $\left./ \mathrm{ml}\right)$ with migDCs $\left(0\right.$ to $3 \times 10^{6} \mathrm{DCs} /$ well $)$. Soluble cytokine expression was similarly measured, also after 72 hours of culture.

Naive OT-II CD4 cells $\left(5 \times 10^{5}\right.$ cells/well) were cultured with purified CD11c $/$ MHC class II ${ }^{\text {hi }}$ lymph node migDCs ( 0 to $2 \times 10^{4} \mathrm{DCs} /$ well) isolated from sensitized mice and OVA $(100 \mu \mathrm{g} / \mathrm{ml})$. In some experiments, murine rIL-12 (0-20 ng/ml; Peprotech, catalog 210-12) was added to the cultures. To assess in vitro priming, culture supernatants were tested for secreted IFN- $\gamma$ by ELISA assay after 72 hours of culture (see below).

Cytokine measurements. Lung protein extracts were prepared by homogenizing tissue in $50 \mathrm{mM}$ Tris$\mathrm{HCl}, \mathrm{pH}$ 7.4, $150 \mathrm{mM} \mathrm{NaCl}, 0.02 \%$ Tween 20, and Complete Mini, EDTA-Free Protease Inhibitor (Roche Applied Science). These extracts were assessed for IFN- $\gamma$, IL-17A, IL-4, IL-5, IL-10, and IL-13 production using a custom-made multiplex assay (Bio-Rad Laboratories). Results were read using a Luminex automated system (Bio-Rad Laboratories).

Cell culture supernatants were assessed for cytokine expression by ELISA assay. DuoSet reagent kits for IFN- $\gamma$ (DY485), IL-5 (DY405), IL-6 (DY406), IL-10 (DY417), IL-13 (DY413), IL-17A (DY421), and IL-12p40 (DY499) were purchased from R\&D Systems. The unlabeled primary antibodies, biotinylated secondary antibodies, and streptavidin-horseradish peroxidase were all used according to the manufacturer's recommendations. Color development was performed using a substrate reagent pack (DY999; R\&D Systems) and was allowed to proceed for 15 minutes. The enzymatic color development reaction was terminated using 2N sulfuric acid (Stop Solution; DY994; R\&D Systems). Absorbance was determined at 450 $\mathrm{nm}$ with optical correction at $570 \mathrm{~nm}$. Cytokine concentration was calculated based on a standard curve generated using cytokine standards included in the DuoSet reagent kits.

Measurement of serum immunoglobulins. At the time of sacrifice, whole blood was collected from individual mice via cardiac puncture. After allowing for clotting at room temperature, the blood was centrifuged at $450 \mathrm{~g}$ for 10 minutes at $25^{\circ} \mathrm{C}$, and then the serum was collected and frozen at $-80^{\circ} \mathrm{C}$. Total serum concentration was measured using Affymetrix eBioscience Ready-SET-Go! kits for IgG1 (catalog 88-50410), IgG2a (catalog 88-50420), and IgE (catalog 88-50460) according to the instructions provided by the manufacturer.

Assays for HDM-specific IgG1 and IgG2a were also developed. Immulon 2 HB plates (Thermo Fisher Scientific, catalog 3455) were coated with $\operatorname{HDM}(10 \mu \mathrm{g} / \mathrm{ml})$ diluted in sterile PBS overnight at $4^{\circ} \mathrm{C}$. Following 3 washes with wash buffer (PBS $+0.05 \%$ Tween 20) the plates were blocked for 1 hour with 200 $\mu 1 /$ well reagent diluent (R\&D Systems, DY995). Serial dilutions of serum test samples, a pooled hyperimmune serum, and pooled serum from untreated animals were prepared in reagent diluent and incubated at $100 \mu \mathrm{l} /$ well for 2 hours at room temperature. The plates were washed 4 times and then incubated 2 hours with HRP-labeled goat anti-mouse IgG1 (A10551; 1:2,000) or IgG2a (M32307; 1:2,000), both from Thermo Fisher Scientific, diluted in reagent diluent at $100 \mu \mathrm{l} /$ well. The plates were washed 4 times and then incubated 20 minutes with streptavidin-horseradish peroxidase (from R\&D Systems DuoSet kits listed above) diluted 1:400 in reagent diluent, $100 \mu 1$ well. After 6 washes, color development was performed using a substrate reagent pack (DY999) and was allowed to proceed for 15 minutes. The enzymatic color development reaction was terminated using $2 \mathrm{~N}$ sulfuric acid (Stop Solution). Absorbance was determined at $450 \mathrm{~nm}$ with optical correction at $570 \mathrm{~nm}$. Representative test curves for HDM-specific IgG2a and IgG1 with immune and naive sera are shown in Supplemental Figure 6A. The absorbance values were compared to the total serum Ig levels obtained above for each sample (Supplemental Figure 6B) and were plotted as arbitrary units (AU). No HDM-specific Igs were ever detected with pooled serum from untreated animals.

$R N A$ isolation and $q R T-P C R$. Lung tissue was homogenized in TRIzol solution (Life Technologies), and lymph node migDCs were lysed by resuspension in RLT buffer (QIAGEN). RNA was prepared from each using an RNeasy kit (QIAGEN). qRT-PCR was performed using TaqMan expression primer and probe sets from Thermo Fisher Scientific as follows: mouse Ifng (Mm01168134_m1), Il4 (Mm00445259_m1), Il5 (Mm004396646_m1), Il6 (Mm00446190_m1), Il10 (Mm01288386_m1), Il12p35 (Mm00434165_m1), Il12p40 (Mm00434174_m1), Il13 (Mm00434204_m1), Irf5 (Mm00496477_m1), Hprt1 (Mm01545399_m1); and human IRF5 (Hs00158114_m1) and HPRT1 (Hs02800695_m1). The reactions were performed at the University of Pittsburgh Genomics Research Core using the ABI PRISM 7700 Sequence System (Applied Biosystems). As an internal reference control, $H p r t 1$ was used to calculate mRNA expression using the $2^{-\Delta C t}$ method. 
Statistics. For paired analysis, the Mann-Whitney $U$ test was routinely employed for nonparametric data; otherwise, the 2-tailed Student's $t$ test was performed. For multiple related comparisons, such as BAL differential counts, 1-way ANOVA with Bonferroni's multiple comparison test was performed. In each case, statistical significance was considered at $P$ less than or equal to $0.05\left({ }^{*} P \leq 0.05 ; * * P \leq 0.01\right.$; $\left.{ }^{* * *} P \leq 0.001 ; * * * * P \leq 0.0001\right)$. All statistical analyses were performed using GraphPad Prism version 5 for OS X software.

Study approval. For human studies that were conducted under the auspices of the SARP, informed consent and samples were obtained according to approved University of Pittsburgh IRB procedures. All mice were bred, housed, and utilized for experimentation according to appropriate and approved University of Pittsburgh IACUC protocols.

\section{Author contributions}

AR conceived the study. TBO, PR, and AR designed the experiments. SEW and MG performed the human subject bronchoscopies and provided the human BAL cells. TBO, MR, CM, REH, RH, MG, SD, KS, and KC performed experiments, and SN helped with statistical analysis of data. TBO and AR analyzed the data and wrote the manuscript.

\section{Acknowledgments}

This work was supported by the NIH grants HL113956, AI106684, and AI048927 (to AR) and AI100012, HL114453, and HL122307 (to PR) and HL69174 (to SEW).

Address correspondence to: Anuradha Ray, NW628 MUH, 3459 Fifth Avenue, Pittsburgh, Pennsylvania 15213, USA. Phone: 412.802.3191; E-mail: raya@pitt.edu.

1. Gauthier M, Ray A, Wenzel SE. Evolving concepts of asthma. Am J Respir Crit Care Med. 2015;192(6):660-668.

2. Ray A, Oriss TB, Wenzel SE. Emerging molecular phenotypes of asthma. Am J Physiol Lung Cell Mol Physiol. 2015;308(2):L130-L140.

3. Ray A, Raundhal M, Oriss TB, Ray P, Wenzel SE. Current concepts of severe asthma. J Clin Invest. 2016;126(7):2394-2403.

4. Chung KF, et al. International ERS/ATS guidelines on definition, evaluation and treatment of severe asthma. Eur Respir J. 2014;43(2):343-373

5. Luyster FS, et al. Sleep quality and asthma control and quality of life in non-severe and severe asthma. Sleep Breath. 2012;16(4):1129-1137.

6. Akinbami LJ, et al. Asthma outcomes: healthcare utilization and costs. J Allergy Clin Immunol. 2012;129(3 Suppl):S49-S64.

7. Barnett SB, Nurmagambetov TA. Costs of asthma in the United States: 2002-2007. J Allergy Clin Immunol. 2011;127(1):145-152.

8. Szefler SJ, et al. Economic burden of impairment in children with severe or difficult-to-treat asthma. Ann Allergy Asthma Immunol. 2011;107(2):110-119.e1.

9. Raundhal M, et al. High IFN- $\gamma$ and low SLPI mark severe asthma in mice and humans. J Clin Invest. 2015;125(8):3037-3050.

10. Leavy O. Asthma and allergy: An IFN $\gamma$ bias in severe asthma. Nat Rev Immunol. 2015;15(8):466-467.

11. Shannon J, et al. Differences in airway cytokine profile in severe asthma compared to moderate asthma. Chest. 2008;133(2):420-426

12. Truyen E, et al. Evaluation of airway inflammation by quantitative Th1/Th2 cytokine mRNA measurement in sputum of asthma patients. Thorax. 2006;61(3):202-208.

13. Voraphani N, et al. An airway epithelial iNOS-DUOX2-thyroid peroxidase metabolome drives Th1/Th2 nitrative stress in human severe asthma. Mucosal Immunol. 2014;7(5):1175-1185.

14. Chambers ES, et al. Distinct endotypes of steroid-resistant asthma characterized by IL-17A(high) and IFN- $\gamma($ high) immunophenotypes: potential benefits of calcitriol. J Allergy Clin Immunol. 2015;136(3):628-637.e4.

15. Borish L, Rosenwasser L. TH1/TH2 lymphocytes: doubt some more. J Allergy Clin Immunol. 1997;99(2):161-164.

16. Guo FH, et al. Interferon gamma and interleukin 4 stimulate prolonged expression of inducible nitric oxide synthase in human airway epithelium through synthesis of soluble mediators. J Clin Invest. 1997;100(4):829-838.

17. Modena $\mathrm{BD}$, et al. Gene expression in relation to exhaled nitric oxide identifies novel asthma phenotypes with unique biomolecular pathways. Am J Respir Crit Care Med. 2014;190(12):1363-1372.

18. Pawliczak R, et al. Influence of IFN-gamma on gene expression in normal human bronchial epithelial cells: modulation of IFN-gamma effects by dexamethasone. Physiol Genomics. 2005;23(1):28-45.

19. Schroder K, Hertzog PJ, Ravasi T, Hume DA. Interferon-gamma: an overview of signals, mechanisms and functions. J Leukoc Biol. 2004;75(2):163-189.

20. Jin FY, Nathan C, Radzioch D, Ding A. Secretory leukocyte protease inhibitor: a macrophage product induced by and antagonistic to bacterial lipopolysaccharide. Cell. 1997;88(3):417-426.

21. Wang Z, et al. Interferon gamma induction of pulmonary emphysema in the adult murine lung. J Exp Med. 2000;192(11):1587-1600

22. Barrios VE, Jarosinski MA, Wright CD. Proteinase-activated receptor-2 mediates hyperresponsiveness in isolated guinea pig bronchi. Biochem Pharmacol. 2003;66(3):519-525.

23. Tam EK, Caughey GH. Degradation of airway neuropeptides by human lung tryptase. Am J Respir Cell Mol Biol. 1990;3(1):27-32.

24. Saliba DG, et al. IRF5:RelA interaction targets inflammatory genes in macrophages. Cell Rep. 2014;8(5):1308-1317. 
25. Krausgruber T, et al. IRF5 promotes inflammatory macrophage polarization and TH1-TH17 responses. Nat Immunol. 2011;12(3):231-238

26. Green BJ, et al. Potentially pathogenic airway bacteria and neutrophilic inflammation in treatment resistant severe asthma. $P L o S$ ONE. 2014;9(6):e100645.

27. Ryjenkov DA, Tarutina M, Moskvin OV, Gomelsky M. Cyclic diguanylate is a ubiquitous signaling molecule in bacteria: insights into biochemistry of the GGDEF protein domain. J Bacteriol. 2005;187(5):1792-1798.

28. Ebensen T, Schulze K, Riese P, Link C, Morr M, Guzmán CA. The bacterial second messenger cyclic diGMP exhibits potent adjuvant properties. Vaccine. 2007;25(8):1464-1469.

29. Ikushima H, Negishi H, Taniguchi T. The IRF family transcription factors at the interface of innate and adaptive immune responses. Cold Spring Harb Symp Quant Biol. 2013;78:105-116.

30. Burdette DL, et al. STING is a direct innate immune sensor of cyclic di-GMP. Nature. 2011;478(7370):515-518.

31. Parvatiyar K, et al. The helicase DDX41 recognizes the bacterial secondary messengers cyclic di-GMP and cyclic di-AMP to activate a type I interferon immune response. Nat Immunol. 2012;13(12):1155-1161.

32. Yin Q, et al. Cyclic di-GMP sensing via the innate immune signaling protein STING. Mol Cell. 2012;46(6):735-745.

33. Barber GN. STING: infection, inflammation and cancer. Nat Rev Immunol. 2015;15(12):760-770.

34. Chesné J, et al. Prime role of IL-17A in neutrophilia and airway smooth muscle contraction in a house dust mite-induced allergic asthma model. J Allergy Clin Immunol. 2015;135(6):1643-1643.e3.

35. Eames HL, Corbin AL, Udalova IA. Interferon regulatory factor 5 in human autoimmunity and murine models of autoimmune disease. Transl Res. 2016;167(1):167-182.

36. Weiss M, et al. IRF5 controls both acute and chronic inflammation. Proc Natl Acad Sci USA. 2015;112(35):11001-11006.

37. Fei M, et al. TNF-alpha from inflammatory dendritic cells (DCs) regulates lung IL-17A/IL-5 levels and neutrophilia versus eosinophilia during persistent fungal infection. Proc Natl Acad Sci USA. 2011;108(13):5360-5365.

38. Oriss TB, et al. Dynamics of dendritic cell phenotype and interactions with $\mathrm{CD} 4^{+} \mathrm{T}$ cells in airway inflammation and tolerance. J Immunol. 2005;174(2):854-863.

39. Krishnamoorthy N, et al. Activation of c-Kit in dendritic cells regulates T helper cell differentiation and allergic asthma. Nat Med. 2008;14(5):565-573.

40. $\mathrm{Xu} \mathrm{H}$, et al. Indoleamine 2,3-dioxygenase in lung dendritic cells promotes Th2 responses and allergic inflammation. Proc Natl Acad Sci USA. 2008;105(18):6690-6695.

41. Cella M, Scheidegger D, Palmer-Lehmann K, Lane P, Lanzavecchia A, Alber G. Ligation of CD40 on dendritic cells triggers production of high levels of interleukin-12 and enhances T cell stimulatory capacity: T-T help via APC activation. J Exp Med. 1996;184(2):747-752.

42. Macatonia SE, et al. Dendritic cells produce IL-12 and direct the development of Th1 cells from naive CD4 ${ }^{+} \mathrm{T}$ cells. $J$ Immunol. 1995;154(10):5071-5079.

43. O'Garra A, Hosken N, Macatonia S, Wenner CA, Murphy K. The role of macrophage- and dendritic cell-derived IL12 in Th1 phenotype development. Res Immunol. 1995;146(7-8):466-472.

44. McGeachy MJ, Cua DJ. Th17 cell differentiation: the long and winding road. Immunity. 2008;28(4):445-453.

45. McGeachy MJ, et al. The interleukin 23 receptor is essential for the terminal differentiation of interleukin 17-producing effector T helper cells in vivo. Nat Immunol. 2009;10(3):314-324.

46. D'Andrea A, et al. Production of natural killer cell stimulatory factor (interleukin 12) by peripheral blood mononuclear cells. J Exp Med. 1992;176(5):1387-1398.

47. Gately MK, et al. The interleukin-12/interleukin-12-receptor system: role in normal and pathologic immune responses. Annu Rev Immunol. 1998;16:495-521.

48. Byrne AJ, et al. A critical role for IRF5 in regulating allergic airway inflammation [published online ahead of print October 19, 2016]. Mucosal Immunol. https://doi.org/10.1038/mi.2016.92.

49. Plantinga $\mathrm{M}$, et al. Conventional and monocyte-derived CD11b(+) dendritic cells initiate and maintain T helper 2 cell-mediated immunity to house dust mite allergen. Immunity. 2013;38(2):322-335.

50. Vermaelen KY, Carro-Muino I, Lambrecht BN, Pauwels RA. Specific migratory dendritic cells rapidly transport antigen from the airways to the thoracic lymph nodes. J Exp Med. 2001;193(1):51-60.

51. Kim TS, Braciale TJ. Respiratory dendritic cell subsets differ in their capacity to support the induction of virus-specific cytotoxic CD8 ${ }^{+}$T cell responses. PLoS ONE. 2009;4(1):e4204

52. Braciale TJ, Sun J, Kim TS. Regulating the adaptive immune response to respiratory virus infection. Nat Rev Immunol. 2012;12(4):295-305

53. Everts B, et al. Migratory $\mathrm{CD} 103^{+}$dendritic cells suppress helminth-driven type 2 immunity through constitutive expression of IL-12. J Exp Med. 2016;213(1):35-51.

54. Ivanov II, et al. The orphan nuclear receptor RORgammat directs the differentiation program of proinflammatory IL-17 ${ }^{+} \mathrm{T}$ helper cells. Cell. 2006;126(6):1121-1133.

55. Lockhart E, Green AM, Flynn JL. IL-17 production is dominated by gammadelta T cells rather than CD4 T cells during Mycobacterium tuberculosis infection. J Immunol. 2006;177(7):4662-4669.

56. Chiaramonte MG, Schopf LR, Neben TY, Cheever AW, Donaldson DD, Wynn TA. IL-13 is a key regulatory cytokine for Th2 cell-mediated pulmonary granuloma formation and IgE responses induced by Schistosoma mansoni eggs. J Immunol. 1999;162(2):920-930

57. Emson CL, Bell SE, Jones A, Wisden W, McKenzie AN. Interleukin (IL)-4-independent induction of immunoglobulin (Ig)E, and perturbation of T cell development in transgenic mice expressing IL-13. J Exp Med. 1998;188(2):399-404.

58. Punnonen J, et al. Interleukin 13 induces interleukin 4-independent IgG4 and IgE synthesis and CD23 expression by human B cells. Proc Natl Acad Sci USA. 1993;90(8):3730-3734.

59. Snapper CM, Peschel C, Paul WE. IFN-gamma stimulates IgG2a secretion by murine B cells stimulated with bacterial lipopolysaccharide. J Immunol. 1988;140(7):2121-2127.

60. Snapper CM, Finkelman FD, Paul WE. Differential regulation of IgG1 and IgE synthesis by interleukin 4. J Exp Med. 
1988;167(1):183-196.

61. Kirby AC, Coles MC, Kaye PM. Alveolar macrophages transport pathogens to lung draining lymph nodes. J Immunol. 2009;183(3):1983-1989.

62. Jang S, Uzelac A, Salgame P. Distinct chemokine and cytokine gene expression pattern of murine dendritic cells and macrophages in response to Mycobacterium tuberculosis infection. J Leukoc Biol. 2008;84(5):1264-1270.

63. Paun A, et al. Functional characterization of murine interferon regulatory factor 5 (IRF-5) and its role in the innate antiviral response. J Biol Chem. 2008;283(21):14295-14308.

64. Khare A, Krishnamoorthy N, Oriss TB, Fei M, Ray P, Ray A. Cutting edge: inhaled antigen upregulates retinaldehyde dehydrogenase in lung $\mathrm{CD}_{103}{ }^{+}$but not plasmacytoid dendritic cells to induce Foxp3 de novo in $\mathrm{CD} 4^{+} \mathrm{T}$ cells and promote airway tolerance. J Immunol. 2013;191(1):25-29.

65. Williams JW, et al. Transcription factor IRF4 drives dendritic cells to promote Th2 differentiation. Nat Commun. 2013;4:2990.

66. Negishi H, et al. Negative regulation of Toll-like-receptor signaling by IRF-4. Proc Natl Acad Sci USA. 2005;102(44):15989-15994.

67. Balzar S, et al. Mast cell phenotype, location, and activation in severe asthma. Data from the Severe Asthma Research Program. Am J Respir Crit Care Med. 2011;183(3):299-309.

68. Yasuda K, et al. Phenotype and function of B cells and dendritic cells from interferon regulatory factor 5-deficient mice with and without a mutation in DOCK2. Int Immunol. 2013;25(5):295-306.

69. Purtha WE, Swiecki M, Colonna M, Diamond MS, Bhattacharya D. Spontaneous mutation of the Dock2 gene in Irf5 $\%$ mice complicates interpretation of type I interferon production and antibody responses. Proc Natl Acad Sci USA. 2012;109(15):E898-E904.

70. Grünig G, et al. Requirement for IL-13 independently of IL-4 in experimental asthma. Science. 1998;282(5397):2261-2263.

71. McMillan SJ, Bishop B, Townsend MJ, McKenzie AN, Lloyd CM. The absence of interleukin 9 does not affect the development of allergen-induced pulmonary inflammation nor airway hyperreactivity. J Exp Med. 2002;195(1):51-57.

72. Ostroukhova M, et al. Tolerance induced by inhaled antigen involves CD4(+) T cells expressing membrane-bound TGF-beta and FOXP3. J Clin Invest. 2004;114(1):28-38.

73. Townsend JM, Fallon GP, Matthews JD, Smith P, Jolin EH, McKenzie NA. IL-9-deficient mice establish fundamental roles for IL-9 in pulmonary mastocytosis and goblet cell hyperplasia but not T cell development. Immunity. 2000;13(4):573-583. 